\title{
Predicting the Mechanical Properties of Viscose/Lycra Knitted Fabrics Using Fuzzy Technique
}

\author{
Ismail Hossain,, ${ }^{1,2}$ Imtiaz Ahmed Choudhury, ${ }^{1}$ Azuddin Bin Mamat, \\ Abdus Shahid, ${ }^{3}$ Ayub Nabi Khan, ${ }^{4}$ and Altab Hossain ${ }^{5}$ \\ ${ }^{1}$ Department of Mechanical Engineering, Faculty of Engineering, University of Malaya, Kuala Lumpur, Malaysia \\ ${ }^{2}$ Department of Textile Engineering, Faculty of Engineering, Daffodil International University, 102 Sukrabad, Mirpur Road, \\ Dhaka 1207, Bangladesh \\ ${ }^{3}$ Department of Textile Engineering, Dhaka University of Engineering and Technology, Gazipur, Bangladesh \\ ${ }^{4}$ BGMEA University of Fashion and Technology (BUFT), Dhaka, Bangladesh \\ ${ }^{5}$ Department of Nuclear Science \& Engineering, Military Institute of Science and Technology, Dhaka, Bangladesh
}

Correspondence should be addressed to Ismail Hossain; ismailpab@yahoo.com

Received 21 November 2015; Accepted 13 April 2016

Academic Editor: Katsuhiro Honda

Copyright (C) 2016 Ismail Hossain et al. This is an open access article distributed under the Creative Commons Attribution License, which permits unrestricted use, distribution, and reproduction in any medium, provided the original work is properly cited.

The main objective of this research is to predict the mechanical properties of viscose/lycra plain knitted fabrics by using fuzzy expert system. In this study, a fuzzy prediction model has been built based on knitting stitch length, yarn count, and yarn tenacity as input variables and fabric mechanical properties specially bursting strength as an output variable. The factors affecting the bursting strength of viscose knitted fabrics are very nonlinear. Hence, it is very challenging for scientists and engineers to create an exact model efficiently by mathematical or statistical model. Alternatively, developing a prediction model via ANN and ANFIS techniques is also difficult and time consuming process due to a large volume of trial data. In this context, fuzzy expert system (FES) is the promising modeling tool in a quality modeling as FES can map effectively in nonlinear domain with minimum experimental data. The model derived in the present study has been validated by experimental data. The mean absolute error and coefficient of determination between the actual bursting strength and that predicted by the fuzzy model were found to be $2.60 \%$ and 0.961 , respectively. The results showed that the developed fuzzy model can be applied effectively for the prediction of fabric mechanical properties.

\section{Introduction}

Knitting is one of the main fabric manufacturing methods among the knitting, weaving, and nonweaving in the textile manufacturing. Basically, knit fabric is formed by intermeshing yarn loops with each other in wale and course directions. The quality of fabrics is considered a big issue in the global textile and apparel market. The demand of knitted fabric especially viscose knitted fabric is increasing rapidly due to their unique quality characteristics such as elasticity, drape, wrinkle resistance, comfort, softness, and easy-care properties over woven fabrics. Viscose knitted fabrics are very popular for apparel wears such as T-shirts, shirts, sweaters, blouses, underwear, casual wear, active wear, and sportswear because of their distinctive quality characteristics as compared to woven fabrics [1-5].

The bursting strength, however, is one of the most important mechanical properties among all the viscose plain knitted fabrics qualities. Knitted fabrics are not just rendering forces in the vertical and perpendicular directions but also they are exposed to multiaxial forces during dyeing, finishing, and usage. Testing tensile and tearing strength in the wale and coarse directions in knitted fabrics are not suitable because of the structural properties; hence, testing bursting strength turns out to be extremely important particularly for knitted fabrics before manufacturing. Generally, the bursting strength test is conducted to evaluate the fabric's capability to withstand multiaxial stresses without breaking off $[1,4,6,7]$. 
Basically, strength of fabrics depends on starting fabric forming materials such as yarn properties and fabric density. The literature review revealed that various studies have been reported on the factors affecting the bursting strength of knitted fabric including yarn type, yarn count, yarn tenacity, yarn breaking elongation, yarn breaking strength, yarn twist, yarn evenness, fabrics GSM, fabric wale and courses, knitting stitch length, cover factor, tightness factors, and relaxation treatment $[1,4,6-8]$.

Moreover, all these factors affecting the bursting strength of knitted fabrics are nonlinear and interactive with other. Hence, it poses a great challenge for scientists and engineers to develop effectively an exact model based on mathematical and statistical techniques $[2,9]$. This is ascribed to the fact that both mathematical and statistical models often fail to capture the nonlinear relationship between inputs and outputs [10].

On the other hand, the intelligent systems such as artificial neural network (ANN) and adaptive neuroinference system (ANFIS) models which have the ability to model in nonlinear domain have been applied by few researchers in the previous research for predicting the bursting strength of knitted fabrics. Jamshaid et al. applied ANFIS model to predict the bursting strength of plain knitted fabrics as a function of yarn tenacity, knitting stitch length, and fabric GSM [1]. Ertuğrul and Uçar applied intelligent technique, namely, ANN and ANFIS models, for the prediction of bursting strength of the knitted fabrics using yarn strength, yarn elongation, and fabric GSM as input variables [6]. Unal et al. presented ANN model for the prediction of bursting strength as a function of yarn strength, yarn count, yarn evenness, yarn twist, yarn elongation, and fabric wales and courses [7]. Bahadir et al. used ANN model for the prediction of bursting strength of knitted fabrics [8]. Zeydan studied ANN model for the prediction of woven fabric strength [11]. However, ANN and ANFIS models need large amounts of noisy input-output data for model parameters optimization, which are challenging, labor intensive, and time consuming processes to collect from the textile knitting industries [1,2, 9]. In addition, ANN does not tell the core logic based on which decisions can be taken [10].

In this regard, fuzzy expert system (FES) is found to be the scientific and engineering solution for quality modeling as FES provides reliable prediction outcome with small experimental data in nonlinear and ill-defined textile domain $[2,9]$. Moreover, a fuzzy model is more reasonable, cheaper in design cost, and much easier to apply in comparison to other models. Furthermore, fuzzy logic is used to solve problems in which descriptions of behavior and observations are imprecise, vague, and uncertain $[2,9,10]$.

The key purpose of this work is to construct fuzzy intelligent model for predicting the bursting strength of viscose/ lycra plain knitted fabrics as a function of knitting stitch length, yarn count, and yarn tenacity, which is not reported in the published literature.

\section{Materials and Method}

2.1. Fuzzy Expert System. The artificial intelligence fuzzy expert system is a structure of multivalued logic and an

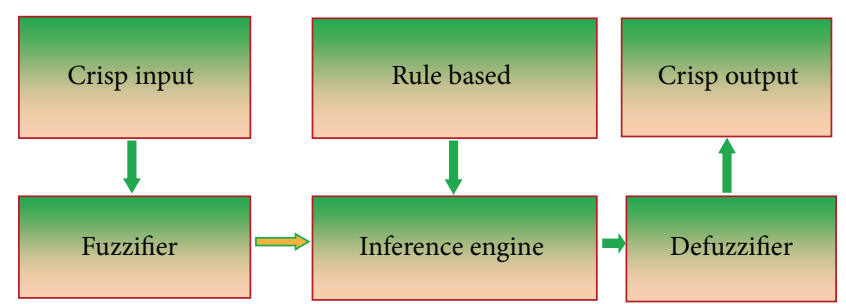

FIgURE 1: Fundamental unit of a fuzzy expert system [2, 15].

extension of crisp logic derived from fuzzy mathematical set theory developed by Zadeh in 1965 [9, 12, 13]. In crisp logic, such as binary logic, variables are true or false, black or white, and 1 or 0 . If the set under investigation is $A$, testing of an element $x$ using the characteristics function $x$ is expressed as follows:

$$
\mu_{A}(x)= \begin{cases}1 ; & \text { if } x \in A \\ 0 ; & \text { if } x \notin A .\end{cases}
$$

A fundamental fuzzy logic unit comprises a fuzzifier, a fuzzy rule base, an inference engine, and a defuzzifier as shown in Figure $1[2,14,15]$.

Fuzzifier. Fuzzifier is the first block in the fuzzy modeling that converts all numeric inputs-outputs into fuzzy sets within a range from 0 to 1 such as low, medium, and high by using membership functions [12]. There are different forms of membership function such as triangle, trapezoid, and Gaussian [16]. Among numerous membership functions, the triangular shaped membership function is the simplest and most frequently applied which is defined as follows [2]:

$$
\mu_{A}(x, a, b, c)= \begin{cases}\frac{x-a}{b-a} ; & a \leq x \leq b \\ \frac{c-x}{c-b} ; & b \leq x \leq c \\ 0 ; & \text { otherwise. }\end{cases}
$$

In this case, the edge of the variable's interval may be represented with linear S-shaped and Z-shaped membership functions described, respectively, as follows.

(i) S-shaped membership functions are

$$
\mu_{\mathrm{S}}(x, a, b)= \begin{cases}1 ; & x \leq a \\ \frac{b-x}{b-a} ; & a \leq x \leq b \\ 0 ; & x \geq b .\end{cases}
$$

(ii) Z-shaped membership functions are

$$
\mu_{\mathrm{Z}}(x, a, b)= \begin{cases}0 ; & x \leq a \\ \frac{x-a}{b-a} ; & a \leq x \leq b \\ 1 ; & x \geq b .\end{cases}
$$


In (2)-(4), $x$ is the input and output variables; $a, b$, and $c$ are the coefficient of membership functions for the explained input and output variables.

The Fuzzy Rule Base. The fuzzy rules which are inferred from expert knowledge and experience are generally articulated by if-then statements that narrate the input variables in the antecedent part and output variables in the consequent part $[2,9]$. Besides, fuzzy rules are the heart of the fuzzy logic expert system which determines the input-output relationship of the model $[9,10]$. The fuzzy rule base can be divided into two classes, namely, the Mamdani and Sugeno [12, 17].

Mamdani Rules. Both of the antecedent and consequence parts are in fuzzy set form.

Sugeno Rules. The antecedent part is in the form of a fuzzy set and the consequence part is made up by a linear equation or constant.

As an expression, when a fuzzy model with two inputs and one output is involved, then development of fuzzy inference rules can be presented as follows.

Mamdani Rule. If $x 1$ is $A 1$ and $x 2$ is $A 2$, then $y$ is $C 1$. Sugeno Rule. If $x 1$ is $A 1$ and $x 2$ is $A 2$, then $y=b 0+$ $b 1 x 1+b 2 \times 2$

where $x 1, x 2$, and $y$ are linguistic variables, $A 1, A 2$, and $C 1$ are the consequent fuzzy numbers that represent the linguistic states, and $b 0, b 1$, and $b 2$ are linear equation parameters.

Inference Engine. The fuzzy inference engine is basically a control mechanism which performs vital role in the fuzzy modeling due to its ability to generate human decision making and deduce control actions by applying the obvious knowledge in the rule base to the task-specific data to appear at some solution or conclusion $[2,18]$. Mamdani suggested the application of a minimum operation rule as a fuzzy inference function. For three-inputs and single-output fuzzy modeling, fuzzy inference method is mathematically expressed as follows [18]:

$$
\begin{aligned}
\alpha_{i} & =\mu_{A_{i}}(\mathrm{SL}) \wedge \mu_{B_{i}}(\mathrm{YC}) \wedge \mu_{C_{i}}(\mathrm{YT}) \\
i=1,2, \ldots n, & \\
\mu_{C}(\mathrm{BS}) & =\bigcup_{i=1}^{n}\left[\alpha_{i} \wedge \mu_{C_{i}}(\mathrm{BS})\right],
\end{aligned}
$$

where $\alpha_{i}$ is the weighting factor as a measure of the contribution of $i$ th rule to the fuzzy control action and $\mu_{A_{i}}, \mu_{B_{i}}, \mu_{C_{i}}$, and $\mu_{C}$ are the membership functions associated with fuzzy sets $A_{i}, B_{i}, C_{i}$, and $C$, respectively.

Defuzzifier. Defuzzifier is the important and last block (unit) of fuzzy modeling. Basically, the conclusion of the inference engine is the fuzzy output. Finally, among different defuzzification methods, the center of gravity method is adopted here to convert the fuzzy inference output into a nonfuzzy value $z$ in the following form for the distinct case $[2,18]$ :

$$
z=\frac{\sum_{i=1}^{n} \mu_{i}\left(b_{i}\right)}{\sum_{i=1}^{n} \mu_{i}},
$$

where $b_{i}$ is the position of the singleton in $i$ th universe and $\mu_{i}$ is the membership function of $i$ rule.

2.2. Prediction Performance Measure. The prediction accuracy of the developed model has been investigated according to the global prediction error such as mean absolute error (MAE) and coefficient of determination $\left(R^{2}\right)$. The formulations of those accuracy measures are given below:

$$
\begin{aligned}
\mathrm{MAE} & =\frac{1}{\mathrm{~N}} \sum_{i=1}^{i=N}\left(\frac{\left|E_{\mathrm{a}}-E_{\mathrm{p}}\right|}{E_{\mathrm{a}}} \times 100\right) \\
R^{2} & =1-\left(\frac{\sum_{i=1}^{i=N}\left(E_{\mathrm{a}}-E_{\mathrm{p}}\right)^{2}}{\sum_{i=1}^{i=N}\left(E_{\mathrm{a}}-E_{\mathrm{M}}\right)^{2}}\right),
\end{aligned}
$$

where $E_{\mathrm{a}}$ is actual result, $E_{\mathrm{p}}$ is predicted result, $E_{\mathrm{M}}$ is mean value, and $N$ is number of patterns.

The coefficient of determinations $\left(R^{2}\right)$ compares the accuracy of the model to that of accuracy of a trivial benchmark model. The mean absolute error (MAE) gives the deviation between the predicted and experimental values and it is required to reach zero [2].

2.3. Development of Fuzzy Prediction Model. For development of fuzzy prediction model, three knitting variables such as knitting stitch length (SL), yarn count (YC), and yarn tenacity (YT) have been used as input variables and bursting strength of knitted fabrics as output variable. These knitting variables have been exclusively selected as they influence the fabric bursting strength considerably. A fuzzy logic Toolbox from MATLAB (version 7.10.0) was used to develop the proposed fuzzy model of bursting strength. The construction of fuzzy modeling for bursting strength has been depicted in Figure 2.

For fuzzification, four possible linguistic subsets, namely, very low (VL), low (L), medium $(\mathrm{M})$, and high $(\mathrm{H})$, for input variables SL and YC and three convenient linguistic subsets, namely, low (L), medium (M), and high (H), for input variable YT were chosen in such a way that they are evenly spaced and cover up the entire input spaces. Ten output fuzzy sets (Levels 1 to 10) (where $L=$ Level) are considered for fabric bursting strength (BS), so that the fuzzy expert system can map small changes in bursting strength with the changes in input variables. In this study, the triangular shaped membership functions are used for input-output variables because of their accuracy. Moreover, a Mamdani max-min inference approach and the center of gravity defuzzification method have been applied in this work. Selection of the number of membership functions and their initial values is based on the system knowledge and experimental conditions [2]. There is a level of membership for each linguistic word 


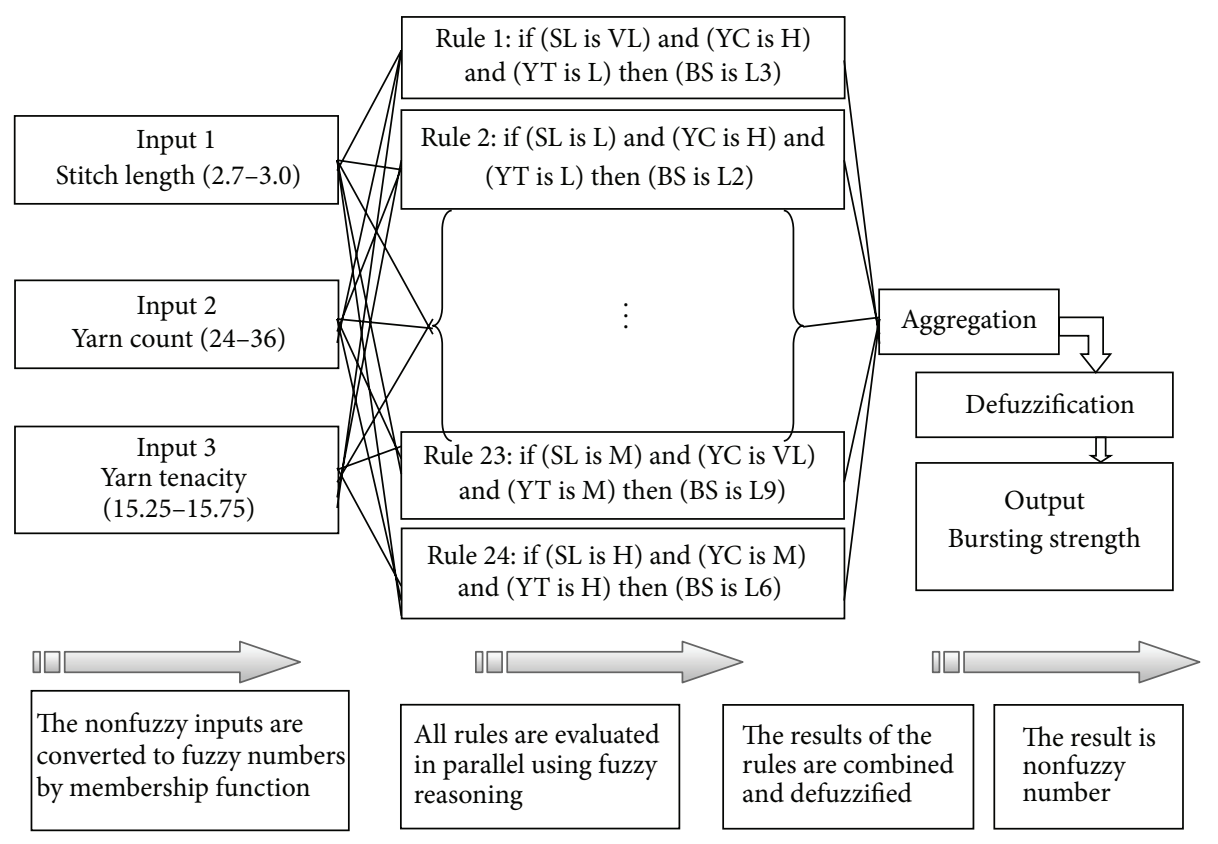

FIGURE 2: Schematic diagram of fuzzy modeling for bursting strength [9].

that applies to that input variable. Fuzzification of the used factors is made with the aid of the following functions:

$$
\begin{aligned}
& \mathrm{SL}\left(i_{1}\right)= \begin{cases}i_{1} ; & 2.7 \leq i_{1} \leq 3.0 \\
0 ; & \text { otherwise }\end{cases} \\
& \mathrm{YC}\left(i_{2}\right)= \begin{cases}i_{2} ; & 24 \leq i_{2} \leq 36 \\
0 ; & \text { otherwise }\end{cases} \\
& \mathrm{YT}\left(i_{3}\right)= \begin{cases}i_{3} ; & 15.25 \leq i_{3} \leq 15.75 \\
0 ; & \text { otherwise }\end{cases} \\
& \operatorname{BS}\left(o_{1}\right)= \begin{cases}o_{1} ; & 270 \leq o_{1} \leq 460 \\
0 ; & \text { otherwise }\end{cases}
\end{aligned}
$$

where $i_{1}, i_{2}$, and $i_{3}$ are the first (SL), second (YC), and third (YT) input variables, respectively, and $o_{1}$ is the output variable (BS) showing in (9).

The triangular shaped membership functions for the fuzzy variables, namely, stitch length (SL), yarn count (YC) and yarn tenacity (YT), and bursting strength (BS), have been created using fuzzy logic Toolbox from MATLAB software (version 7.10.0) and are shown in Figures 3-6.

The coefficients of membership functions for the fuzzy inference system (FIS) parameters are shown in Table 1.

Conceptually, there could be $4 \times 4 \times 3=48$ fuzzy rules, as input variable SL having 4 linguistic levels, YC having 4 linguistic levels, and YT having 3 linguistic levels. However, in order to make the fuzzy expert system simpler, only 24 fuzzy rules have been formed based on expert knowledge and

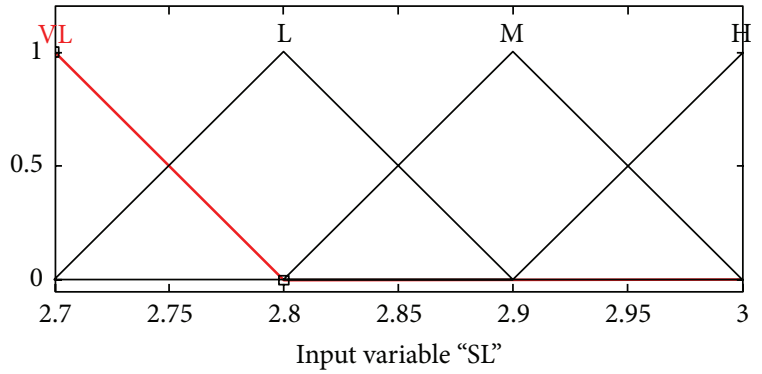

Figure 3: Membership function of input variable "SL."

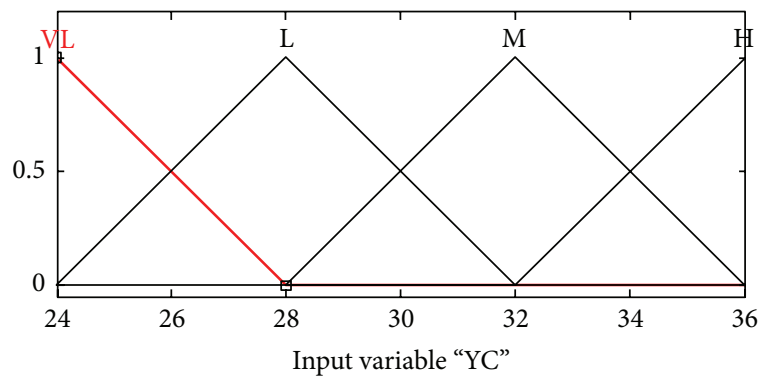

FIgURE 4: Membership function of input variable "YC."

previous experience [9]. Some of the rules are presented in Table 2.

To demonstrate the fuzzification process, linguistic expressions and membership functions of stitch length (SL), yarn count (YC), and yarn tenacity (YT) obtained from 


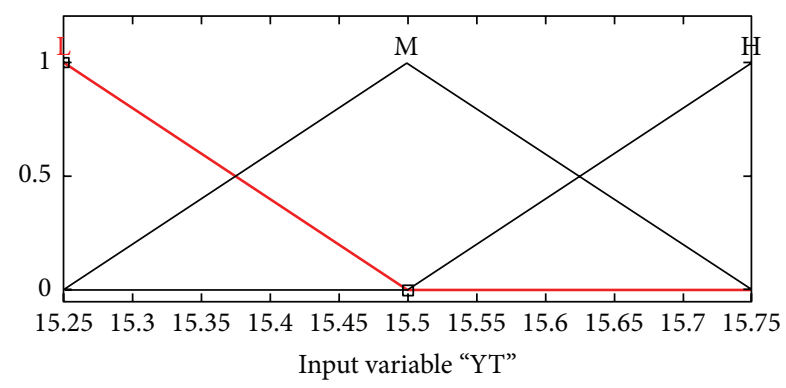

FIgURE 5: Membership function of input variable "YT."

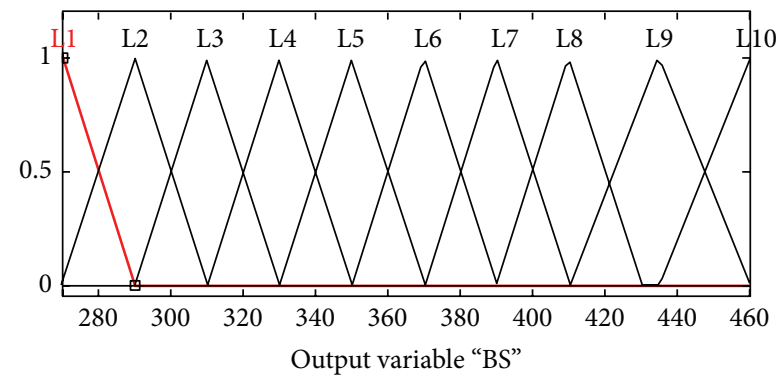

Figure 6: Membership function of output variable "BS."

TABLE 1: Coefficients of membership functions for FIS parameter of BS.

\begin{tabular}{lcccc}
\hline \multirow{2}{*}{ Linguistic variables } & Type & \multicolumn{3}{c}{ Coefficients (\%) } \\
& & $a$ & $b$ & $c$ \\
\hline Level 1 & Z-shaped & 250 & 270 & - \\
Level 2 & Triangular & 250 & 270 & 290 \\
Level 3 & Triangular & 270 & 290 & 310 \\
Level 4 & Triangular & 290 & 310 & 330 \\
Level 5 & Triangular & 310 & 330 & 350 \\
Level 6 & Triangular & 330 & 350 & 370 \\
Level 7 & Triangular & 350 & 370 & 390 \\
Level 8 & Triangular & 370 & 390 & 410 \\
Level 9 & Triangular & 390 & 410 & 435 \\
Level 10 & Triangular & 410 & 435 & 460 \\
\hline
\end{tabular}

TABLE 2: Fuzzy inference rules.

\begin{tabular}{lcccc}
\hline \multirow{2}{*}{ Rules number } & \multicolumn{3}{c}{ Input variables } & Output variable \\
& SL & YC & YT & BS \\
\hline 1 & VL & H & L & Level 3 \\
2 & L & H & L & Level 2 \\
$\vdots$ & $\vdots$ & & $\vdots$ & $\vdots$ \\
10 & L & L & M & Level 5 \\
11 & M & L & M & Level 6 \\
$\vdots$ & $\vdots$ & & $\vdots$ & $\vdots$ \\
23 & M & VL & M & Level 9 \\
24 & H & M & H & Level 6 \\
\hline
\end{tabular}

the developed rules and above formula (9) are presented as follows:

$$
\begin{aligned}
& \mu_{L}(\mathrm{SL})= \begin{cases}\frac{i_{1}-2.7}{2.8-2.7} ; & 2.7 \leq i_{1} \leq 2.8 \\
\frac{2.9-i_{1}}{2.9-2.8} ; & 2.8 \leq i_{1} \leq 2.9 \\
0 ; & i_{1} \geq 2.9\end{cases} \\
& \mu_{L}(\mathrm{SL})=\left\{\frac{0}{2.7}+\frac{0.05}{2.75}+\frac{1}{2.8}+\cdots+\frac{0.05}{2.85}+\frac{0}{2.9}\right\} \\
& \mu_{L}(\mathrm{YC})= \begin{cases}\frac{i_{2}-24}{28-24} ; & 24 \leq i_{2} \leq 28 \\
\frac{32-i_{2}}{32-28} ; & 28 \leq i_{2} \leq 32 \\
0 ; & i_{2} \geq 32\end{cases} \\
& \mu_{L}(\mathrm{YC})=\left\{\frac{0}{24}+\frac{0.5}{26}+\frac{1}{28}+\cdots+\frac{0.5}{30}+\frac{0}{32}\right\} \\
& \mu_{M}(\mathrm{YT})= \begin{cases}\frac{i_{3}-15.25}{15.5-15.25} ; & 15.25 \leq i_{3} \leq 15.5 \\
\frac{15.75-i_{3}}{15.75-15.5} ; & 15.5 \leq i_{3} \leq 15.75 \\
0 ; & i_{3} \geq 15.75\end{cases}
\end{aligned}
$$

$$
\begin{aligned}
& \mu_{M}(\mathrm{YT}) \\
& =\left\{\frac{0}{15.25}+\frac{0.04}{15.35}+\frac{1}{15.5}+\cdots+\frac{0.6}{15.65}+\frac{0}{15.75}\right\} .
\end{aligned}
$$

In defuzzification stage, the membership functions $(\mu)$ of the rules are calculated for each rule by (3). To comprehend fuzzification, an example is considered. For crisp input SL = $2.8 \mathrm{~mm}, \mathrm{YC}=28 \mathrm{Ne}$, and $\mathrm{YT}=15.5 \mathrm{~g} / \mathrm{tex}$, the rule 10 is fired. The firing weighting factor $\alpha$ of the one rule is calculated by (4) and found as

$$
\begin{aligned}
\alpha_{10} & =\min \left\{\left(\mu_{L}(\mathrm{SL}), \mu_{L}(\mathrm{YC}), \mu_{M}(\mathrm{YT})\right)\right\} \\
& =\min (1,1,1)=1 .
\end{aligned}
$$

Consequently, according to (5), the membership function for the conclusion reached by rules 10 is obtained as follows:

$$
\mu_{10}(\mathrm{BS})=\min \left\{1, \mu_{L 5}(\mathrm{BS})\right\} \text {. }
$$

Haghighat et al. have cited that, in many circumstances, for a system whose output is a fuzzy set, it is essential to aggregate the fuzzy sets into a single fuzzy set by aggregation method [12]. Finally, using (7) and (14) with Table 1 and Figure 6, the crisp output of bursting strength is found to be $330 \mathrm{kPa}$.

\section{Experimental Work for the Validation of Fuzzy Model}

3.1. Fabric Knitting and Heat Setting. In this research, total 12 viscose/lycra blended plain fabrics samples were knitted according to Table 3 knitted fabric variables on Pailung single 
TABLE 3: Knitted fabric variables and their level.

\begin{tabular}{lccccc}
\hline \multirow{2}{*}{ Process parameters } & \multirow{2}{*}{ Unit } & \multicolumn{4}{c}{ Level } \\
& & 1 & 2 & 3 & 4 \\
\hline Stitch length & $\mathrm{mm}$ & 2.7 & 2.8 & 2.9 & 3.0 \\
Yarn count & $\mathrm{Ne}$ & 24 & 30 & 34 & \\
Yarn tenacity & $\mathrm{g} / \mathrm{tex}$ & 15.25 & 15.5 & 15.75 & \\
\hline
\end{tabular}

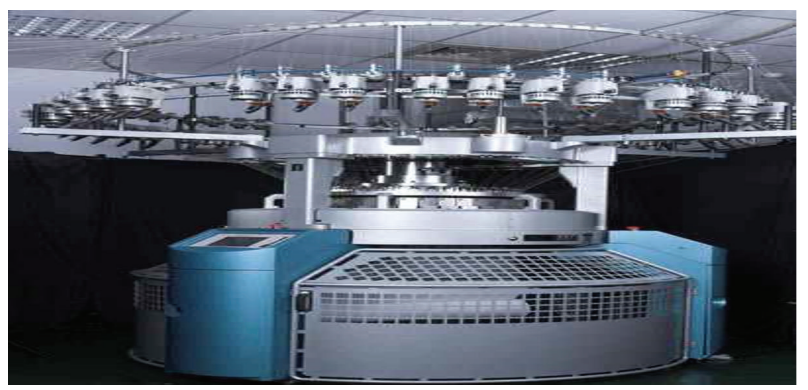

Figure 7: Circular knitting machine (APS Textile, Bangladesh).

jersey circular knitting machine (Figure 7), having 30 inches diameter, 20 gauges (needles/inch), and 90 yarn feeders. The jersey knitted fabric was prewetted on the padding mangle using $2 \mathrm{~g} / \mathrm{L}$ wetting detergent (Feloson NOF) and $1 \mathrm{~g} / \mathrm{L}$ lubricating agent (Kappavon CL $1 \mathrm{~g} / \mathrm{L}$ ) and then heat setting was conducted on the pin stenter finishing machine at a temperature of $200^{\circ} \mathrm{C}$ for 45 seconds of curing time.

3.2. Fabric Processing and Testing. The fabrics samples were subjected to semibleached at $90^{\circ} \mathrm{C}$ for $40 \mathrm{~min}$ in a sample dyeing machine using anticreasing agent (Kappavon CL $1 \mathrm{~g} / \mathrm{L}$ ), sequestering agent (Sirrix $2 \mathrm{UD} 0.5 \mathrm{~g} / \mathrm{L}$ ), wetting agent (Felosan NOF $1 \mathrm{~g} / \mathrm{L})$, soda ash $(2.5 \mathrm{~g} / \mathrm{L})$, hydrogen peroxide $50 \%(1 \mathrm{~g} / \mathrm{L})$, and stabilizing agent $(0.2 \mathrm{~g} / \mathrm{L})$. Then the fabrics samples were washed with proper rinsing and finally treated with acetic acid $(1 \mathrm{~g} / \mathrm{L})$ and peroxide killing agent $(0.2 \mathrm{~g} / \mathrm{L})$ for neutralizing and peroxide killing, respectively. After bleaching, the fabric samples were dried in an open stenter and compacted properly. After production, all the fabrics samples were conditioned on a flat surface first for at least 24 hours prior to testing under standard atmospheric conditions at relative humidity $(65 \pm 2) \%$ and temperature $(20 \pm 2)^{\circ} \mathrm{C}$. Then the bursting strength $(\mathrm{kPa})$ of each sample was tested using SDL ATLAS Pneumatic Bursting tester (Model 229P) with a specimen of $30 \mathrm{~mm}$ in diameter according to ISO139388-1 test method.

\section{Results and Discussion}

4.1. Analysis of Model Performance. The graphical operation of the fuzzy prediction model has been depicted with an example in Figure 8. For simple expression, only one fuzzy rule out of twenty four has been shown in the picture. As per this rule, if knitting stitch length (SL) is low (L), yarn count (YC) is low (L), and yarn tenacity (YT) is medium (M) then output fabric bursting strength (BS) will be Level

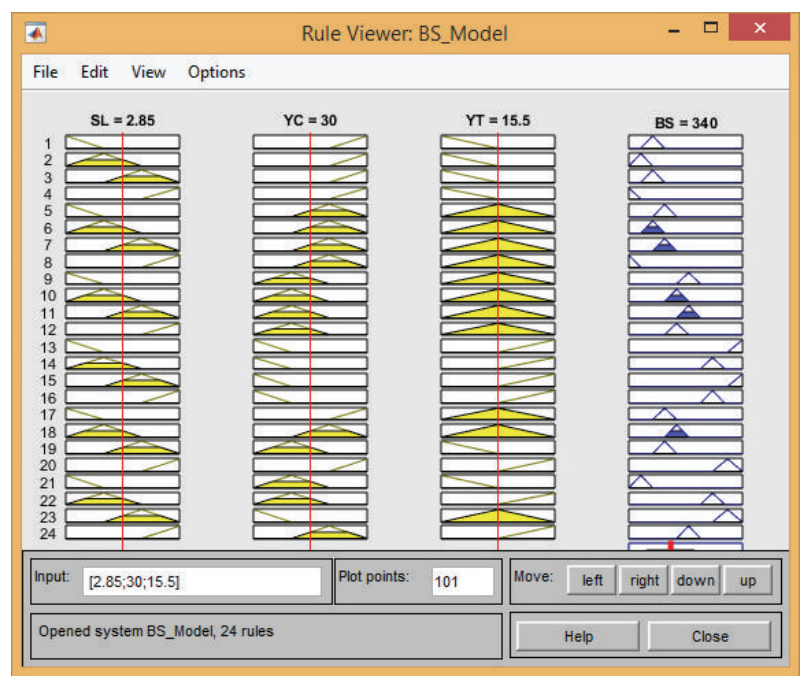

FIGURE 8: Rule viewer.

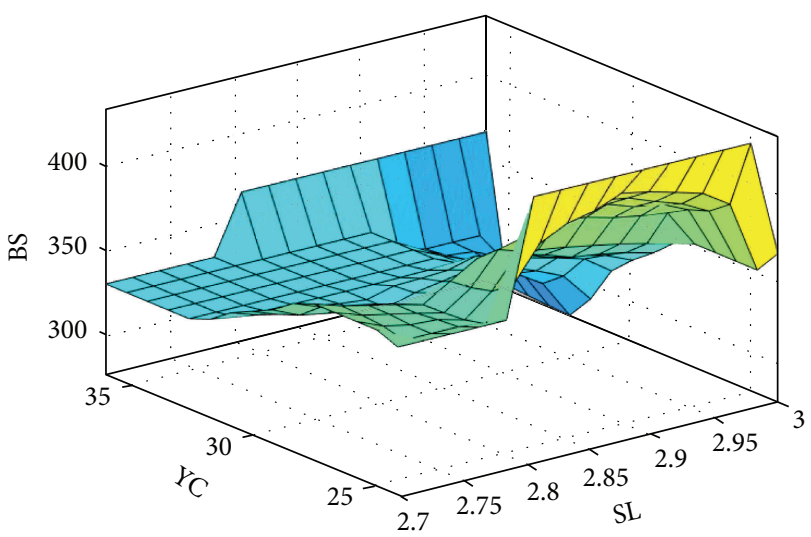

FIGURE 9: Surface plot showing the impact of stitch length and yarn count on bursting strength.

5 (L5). For example, if input SL is $2.8 \mathrm{~mm}, \mathrm{YC}$ is $28 \mathrm{Ne}$, and YT is $15.5 \mathrm{~g} /$ tex, then fuzzy expert model predicted output BS is $330 \mathrm{kPa}$. Using MATLAB Fuzzy Toolbox the fuzzy control surface was developed as shown in Figures 9 and 10. These can serve as a visual depiction of how the fuzzy expert system operates dynamically over time. The images show the relationship between knitting stitch length (SL), yarn count (YC), and yarn tenacity (YT) on the input side and bursting strength (BS) on the output side. The surface plots shown in Figures 9 and 10 depict the impact of stitch length, yarn count, and yarn tenacity on bursting strength.

4.2. Analysis of Experimental Results. Effects of stitch length, yarn count, and yarn tenacity on bursting strength have been shown in Figures 11 and 12. It is obvious from Figure 11 that decrease in knitting stitch length from $3.0 \mathrm{~mm}$ to $2.7 \mathrm{~mm}$ only slightly increases the fabric bursting strength. Approximately, bursting strength increases $15-20 \%$ with a decrease of $10 \%$ in stitch length. The reason for an increase in bursting strength with a decrease in stitch length is probably due to increasing number of loops per unit area which bears 


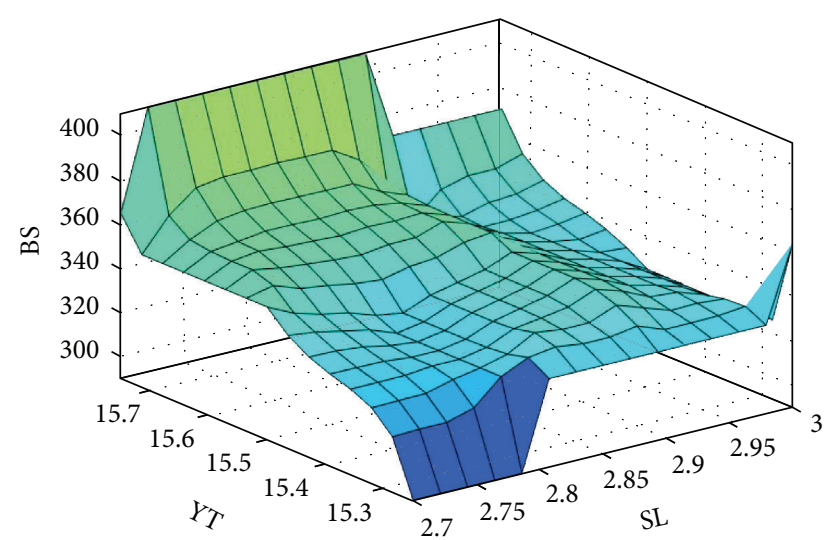

FIGURE 10: Surface plot showing the impact of stitch length and yarn tenacity on bursting strength.

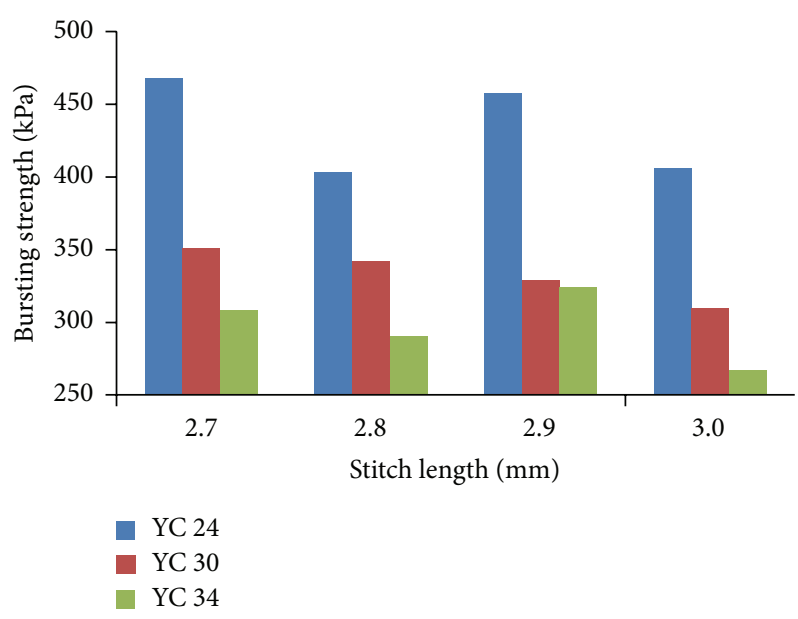

FIGURE 11: Effect of stitch length and yarn count on bursting strength.

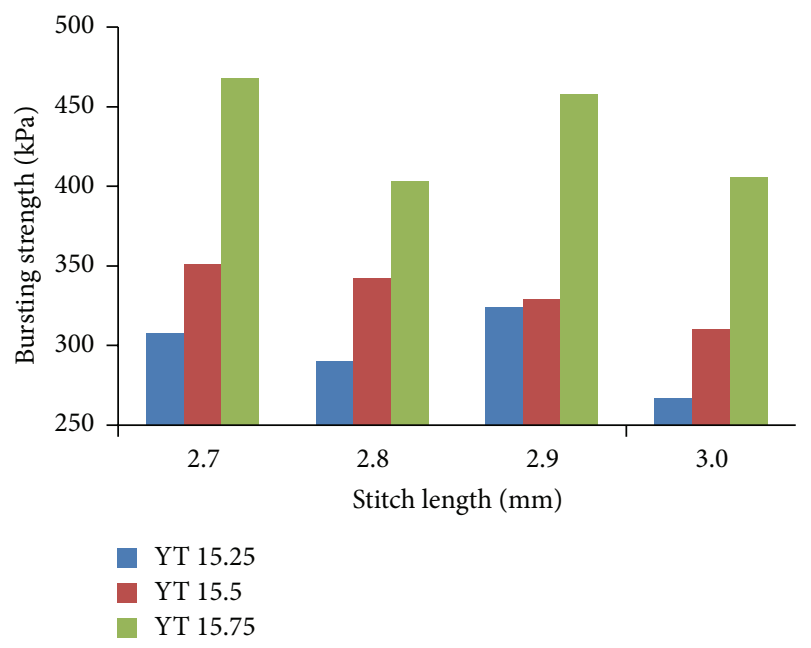

FIGURE 12: Effect of stitch length and yarn tenacity on bursting strength. the multidirectional forces. On the other hand, the effect of decreasing the stitch length is not linear; as a consequence, while the stitch length decreases further than an optimal level, produced fabric turns more stiffer and less extensible, hence resulting in fabric holes as well as poor bursting strength.

A similar phenomenon has been observed for yarn count on bursting strength as shown in Figure 11. It shows that fabric bursting strength increases a little with the decrease of yarn count and vice versa. The bursting strength increases 30-40\% with a decrease of $30 \%$ in yarn count due to the increasing of yarn linear density which would result in increase in bursting strength. Since effect of yarn count on bursting strength is not linear, as a result, produced fabric turns much stiffer and less extensible, while the yarn count decreases with a lower stitch length more than an optimal point and hence leads to poor bursting strength.

In contrast, the effect of yarn tenacity on the bursting strength is much more reflective as compared to stitch length as shown in Figure 12. The figure shows that fabric bursting strength increases drastically with the increase in yarn tenacity and vice versa. The bursting strength increases by about $40-50 \%$ with the increase of $3 \%$ in yarn tenacity. Basically, yarn is the main material for fabrics and yarn tenacity indicates the yarn strength; thus, when yarn tenacity increases, fabric strength increases. However, it was found from Figure 12 that knitting stitch length and yarn tenacity have strong effect on fabric bursting strength. The extensibility of the fabric decreases whilst the yarn tenacity is increased further at lower stitch length levels and as a result, the bursting strength exhibits a descending tendency.

From this investigation, it is clearly observed that yarn tenacity has the greatest and main effect on bursting strength as compared to knitting stitch length and yarn count. Therefore, it is very important to maintain optimum level of knitting parameter in the knitting process in order to achieve the required bursting strength with good quality fabrics.

4.3. Validation of Fuzzy Prediction Model. The developed fuzzy prediction model has been validated by experimental data. Prediction was done using the fuzzy logic rule viewer. The results from the developed fuzzy model were then compared with 12 validated experimental results as shown in Table 4 . The correlations between predicted values and experimental values of fabric bursting strength are also represented in Figure 13. The coefficient of determination $\left(R^{2}\right)$ between the experimental bursting strength and that predicted by the fuzzy model was found to be 0.961 . Therefore, it can be concluded that the developed fuzzy model can explain up to $96.1 \%$ of the total variability of fabric bursting strength. The mean absolute error (MAE) between the actual values and the predicted values of bursting strength was found to be $2.6 \%$ $(<5 \%)$. The absolute error gives the deviation between the predicted and experimental values and it is required to reach towards zero. The results of the coefficient of determination $\left(R^{2}\right)$ and mean absolute error (MAE)\% indicate that the developed fuzzy model has very strong prediction ability with a great accuracy. 
TABLE 4: Comparison of predicted and experimental values of fabric bursting strength.

\begin{tabular}{|c|c|c|c|c|c|c|}
\hline Number & $\begin{array}{l}\text { Stitch length } \\
(\mathrm{mm})\end{array}$ & $\begin{array}{l}\text { Yarn count } \\
(\mathrm{Ne})\end{array}$ & $\begin{array}{c}\text { Yarn tenacity } \\
(\mathrm{g} / \mathrm{tex})\end{array}$ & $\begin{array}{c}\text { Predicted } \\
\text { bursting strength }\end{array}$ & $\begin{array}{c}\text { Experimental } \\
\text { bursting strength }\end{array}$ & $\begin{array}{c}\text { Absolute error } \\
(\%)\end{array}$ \\
\hline 1 & 2.7 & 34 & 15.25 & 310 & 308 & 0.65 \\
\hline 2 & 2.8 & 34 & 15.25 & 290 & 290 & 0.00 \\
\hline 3 & 2.9 & 34 & 15.25 & 310 & 324 & 4.32 \\
\hline 4 & 3 & 34 & 15.25 & 278 & 267 & 4.12 \\
\hline 5 & 2.7 & 30 & 15.5 & 350 & 351 & 0.28 \\
\hline 6 & 2.8 & 30 & 15.5 & 330 & 342 & 3.51 \\
\hline 7 & 2.9 & 30 & 15.5 & 350 & 329 & 6.38 \\
\hline 8 & 3 & 30 & 15.5 & 324 & 310 & 4.52 \\
\hline 9 & 2.7 & 24 & 15.75 & 452 & 468 & 3.42 \\
\hline 10 & 2.8 & 24 & 15.75 & 410 & 403 & 1.74 \\
\hline 11 & 2.9 & 24 & 15.75 & 452 & 458 & 1.31 \\
\hline 12 & 3 & 24 & 15.75 & 410 & 406 & 0.99 \\
\hline & \multicolumn{4}{|c|}{ Mean absolute error (\%) } & \multicolumn{2}{|c|}{2.60} \\
\hline & \multicolumn{4}{|c|}{ Coefficient of determination $\left(R^{2}\right)$} & \multicolumn{2}{|c|}{0.961} \\
\hline
\end{tabular}

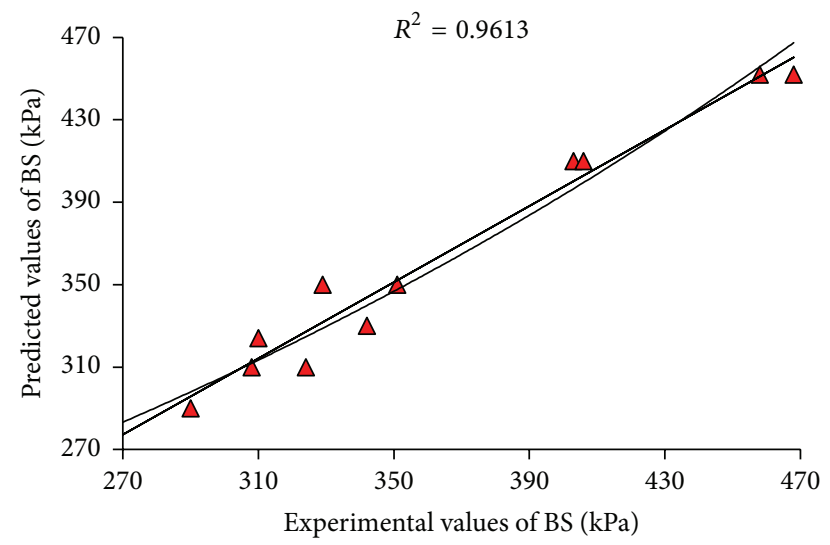

Figure 13: Correlation between experimental and fuzzy model predicted values of bursting strength.

\section{Conclusions}

In this research investigation, fuzzy model has been developed for predicting the fabric mechanical properties like bursting strength of viscose/lycra plain knitted fabric. The prediction model was made by taking the knitting stitch length, yarn count, and yarn tenacity as input variables and fabric bursting strength as output variable. The developed prediction model confers an excellent understanding about the interaction between knitting process variables and their effects on the fabric bursting strength. From the experimental study, it has been found that yarn tenacity has the greatest and main effects on the fabric bursting strength than that of yarn count and knitting stitch length. The fuzzy model derived in this research has been verified from the experiment data.

The mean absolute error (MAE) and coefficient of determination $\left(R^{2}\right)$ between the experimental bursting strength and that predicted by the fuzzy model were found to be $2.6 \%$ and 0.961 , respectively. The results exhibit an excellent prediction performance of the developed fuzzy model. Hence, it can be decisively concluded that the developed fuzzy model can be applied in the textile industry as a decision making support tool for the production engineer to predict the mechanical properties of viscose knitted fabrics satisfactorily.

\section{Competing Interests}

The authors declare that there is no conflict of interests regarding the publication of this paper.

\section{Acknowledgments}

The authors are thankful to the management of textile research laboratory of APS and APS Textile, APS Group Bangladesh for providing the facilities for this research work.

\section{References}

[1] H. Jamshaid, T. Hussain, and Z. A. Malik, "Comparison of regression and adaptive neuro-fuzzy models for predicting the bursting strength of plain knitted fabrics," Fibers and Polymers, vol. 14, no. 7, pp. 1203-1207, 2013.

[2] I. Hossain, A. Hossain, and I. A. Choudhury, "Color strength modeling of viscose/Lycra blended fabrics using a fuzzy logic approach," Journal of Engineered Fibers and Fabrics, vol. 10, no. 1, pp. 158-168, 2015.

[3] I. Hossain, A. Hossain, I. A. Choudhury, A. Bakar, and H. Uddin, "Prediction of fabric properties of viscose blended knitted fabrics by fuzzy logic methodology," in Proceedings of the International Conference on Mechanical and Civil and Architectural Engineering 2014, (ICMCAE '14), (IISRO '14), pp. 100106, Kuala Lumpur, Malaysia, February 2014.

[4] S. Mavruz and R. T. Ogulata, "Taguchi approach for the optimisation of the bursting strength of knitted fabrics," FIBERS \& TEXTILES in Eastern Europe, vol. 79, no. 2, pp. 78-83, 2010. 
[5] T. Shaikh, S. Chaudhari, and A. Varma, "Viscose rayon: a legendary development in the man made textile," International Journal of Engineering Research and Application, vol. 2, no. 5, pp. 675-680, 2012.

[6] S. Ertuğrul and N. Uçar, "Predicting bursting strength of cotton plain knitted fabrics using intelligent techniques," Textile Research Journal, vol. 70, no. 10, pp. 845-851, 2000.

[7] P. G. Unal, M. E. Üreyen, and D. Mecit, "Predicting properties of single jersey fabrics using regression and artificial neural network models," Fibers and Polymers, vol. 13, no. 1, pp. 87-95, 2012.

[8] M. C. Bahadir, S. K. Bahadir, and F. Kalaoglu, "An artificial neural network model for prediction of bursting strength of knitted fabrics," in Proceedings of the International Conference on Machine Learning and Computer Science (IMLCS '12), pp. 167170, August 2012.

[9] A. Majumdar and A. Ghosh, "Yarn strenghth modeling using fuzzy expert system," Journal of Engineered Fibers and Fabrics, vol. 3, no. 4, pp. 61-68, 2008.

[10] P. Hatua, A. Majumdar, and A. Das, "Modeling ultraviolet protection factor of polyester-cotton blended woven fabrics using soft computing approaches," Journal of Engineered Fibers and Fabrics, vol. 9, no. 3, pp. 99-106, 2014.

[11] M. Zeydan, "Modelling the woven fabric strength using artificial neural network and Taguchi methodologies," International Journal of Clothing Science and Technology, vol. 20, no. 2, pp. 104-118, 2008.

[12] E. Haghighat, S. S. Najar, and S. M. Etrati, "The prediction of needle penetration force in woven denim fabrics using soft computing models," Journal of Engineered Fibers and Fabrics, vol. 9, no. 4, pp. 45-55, 2014.

[13] L. A. Zadeh, "Fuzzy sets," Information and Computation, vol. 8, pp. 338-353, 1965.

[14] K. M. U. Darain, M. Z. Jumaat, M. A. Hossain et al., "Automated serviceability prediction of NSM strengthened structure using a fuzzy logic expert system," Expert Systems with Applications, vol. 42, no. 1, pp. 376-389, 2015.

[15] M. Gopal, Digital Control and State Variable Methods: Conventional and Intelligent Control Systems, Tata McGraw-Hill Education Pvt. Ltd, Singapore, 3rd edition, 2009.

[16] M. Verma and K. K. Shukla, "Application of fuzzy optimization to the orienteering problem," Advances in Fuzzy Systems, vol. 2015, Article ID 569248, 12 pages, 2015.

[17] M. Vadood, "Predicting the color index of acrylic fiber using fuzzy-genetic approach," The Journal of the Textile Institute, vol. 105, no. 7, pp. 779-788, 2014.

[18] C.-C. Huang and W.-H. Yu, "Control of dye concentration, $\mathrm{pH}$, and temperature in dyeing processes," Textile Research Journal, vol. 69, no. 12, pp. 914-918, 1999. 

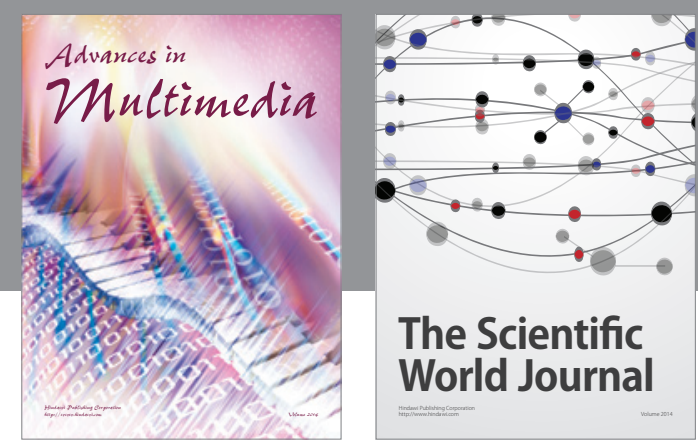

The Scientific World Journal
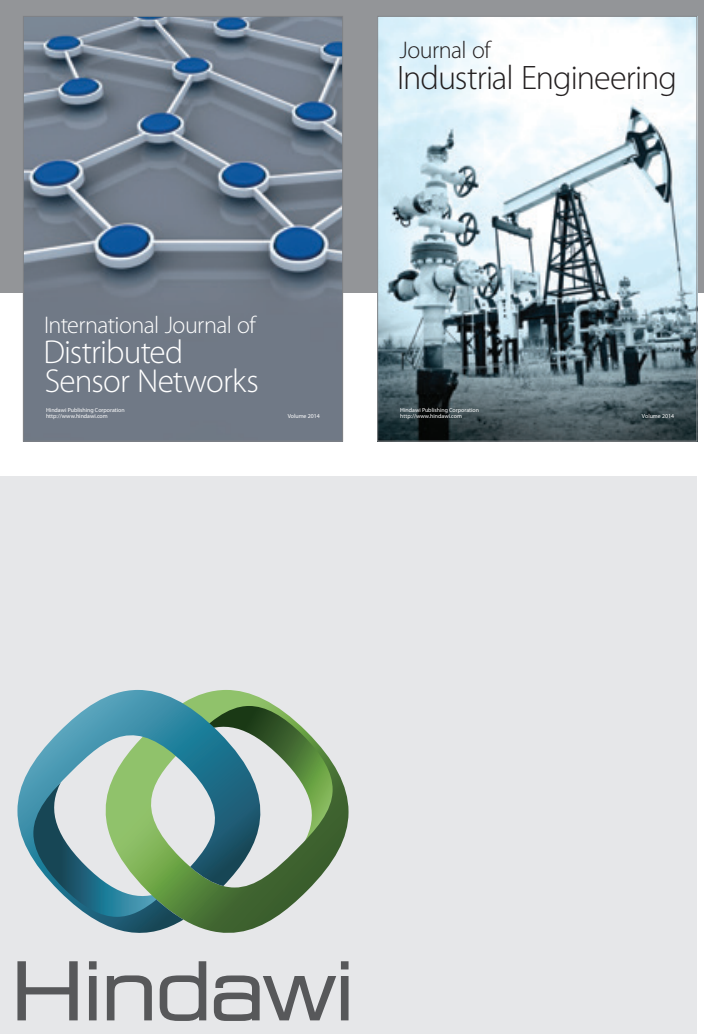

Submit your manuscripts at

http://www.hindawi.com

\section{Computer Networks} and Communications
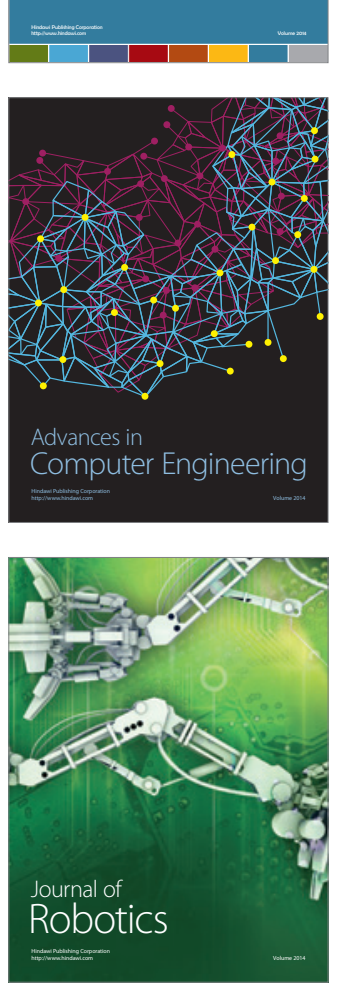
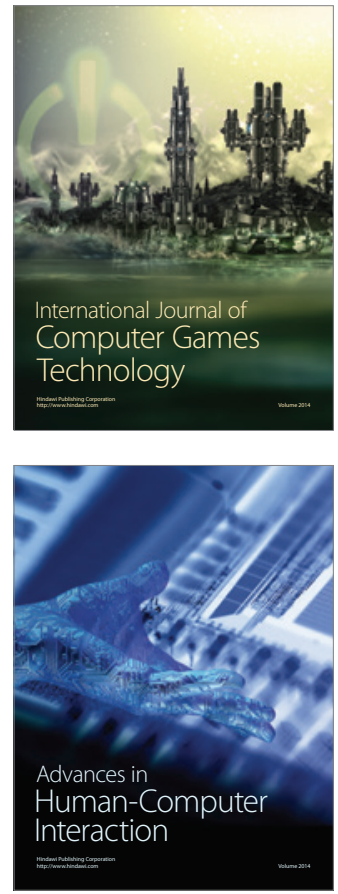
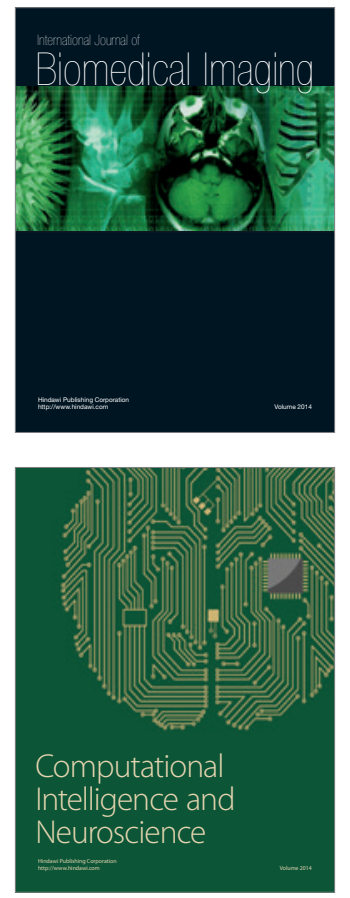
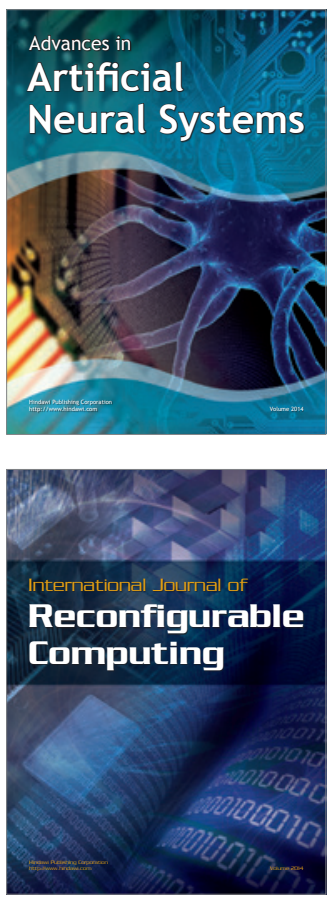
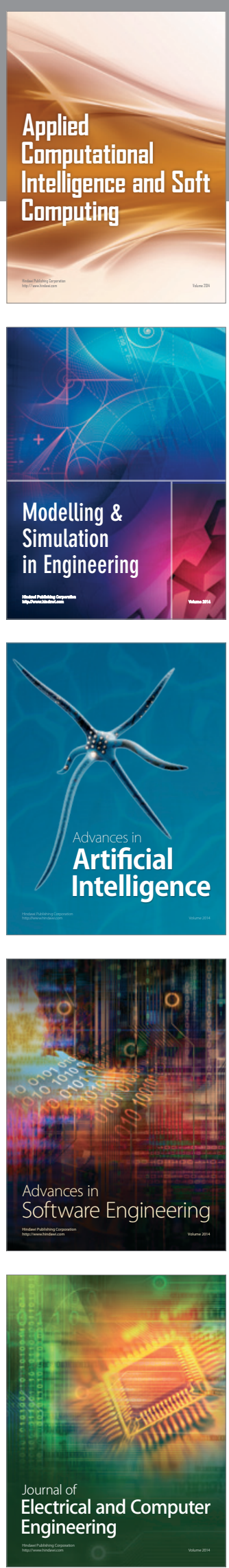